\title{
Mimusops elengi bark extract mediated green synthesis of gold nanoparticles and study of its catalytic activity
}

\author{
Rakhi Majumdar ${ }^{1} \cdot$ Braja Gopal Bag $^{1} \cdot$ Pooja Ghosh $^{1}$
}

Received: 6 March 2015/Accepted: 27 April 2015/Published online: 20 May 2015

(c) The Author(s) 2015. This article is published with open access at Springerlink.com

\begin{abstract}
The bark extract of Mimusops elengi is rich in different types of plant secondary metabolites such as flavonoids, tannins, triterpenoids and saponins. The present study shows the usefulness of the bark extract of Mimusops elengi for the green synthesis of gold nanoparticles in water at room temperature under very mild conditions. The synthesis of the gold nanoparticles was complete within a few minutes without any extra stabilizing or capping agents and the polyphenols present in the bark extract acted as both reducing as well as stabilizing agents. The synthesized colloidal gold nanoparticles were characterized by HRTEM, surface plasmon resonance spectroscopy and $\mathrm{X}$-ray diffraction studies. The synthesized gold nanoparticles have been used as an efficient catalyst for the reduction of 3-nitrophenol and 4-nitrophenol to their corresponding aminophenols in water at room temperature.
\end{abstract}

Keywords Gold nanoparticle - Green synthesis · Mimusops elengi · Polyphenols · Catalytic reduction · Nitrophenol

Electronic supplementary material The online version of this article (doi:10.1007/s13204-015-0454-2) contains supplementary material, which is available to authorized users.

Braja Gopal Bag

braja@mail.vidyasagar.ac.in

1 Department of Chemistry and Chemical Technology, Vidyasagar University, Midnapore 721102, West Bengal, India

\section{Introduction}

Colloidal gold nanoparticles (AuNPs) has been the subject of tremendous research interest in recent years because of their applications in diversified areas such as catalysis, biotechnology, drug delivery, medicine and electronics. (Alkilany et al. 2013; Zhang et al. 2012; Murphy et al. 2008; Prati and Villa 2014; Thomas and Kamat 2003). Due to the large surface to volume ratio, the AuNPs have different physicochemical properties compared to the bulk solids. AuNPs exhibit different colors depending upon their size, shape and degree of aggregation (Weisbecker et al. 1996; Fujiwara et al. 1999; Aslan and Perez-Luna 2002; Mie 1908). The colloidal AuNPs stabilized with non-toxic biomolecules in aqueous medium are required for many of its applications. Among various synthetic methods, plant extract mediated solution phase synthesis of AuNPs involving reduction of $\mathrm{Au}(\mathrm{III})$ to $\mathrm{Au}(0)$ by plant extracts has gained profound significance in recent years because of the renewable and biocompatible nature of the plant extracts, eco-friendly aqueous medium and mild reaction conditions (Anastas and Kirchhoff 2002; Iravani 2011). Moreover, since the plant extract itself acts as a stabilizer, it makes the method more advantageous over other synthetic methods. The extracts of Acacia nilotica leaf (Majumdar and Bag 2013), Lantana camara leaf (Dash et al. 2014a), Abroma augusta Linn bark (Das et al. 2014), Breynia rhamnoides (Gangula et al. 2011), Saraca indica (Dash et al. 2014b), Piper betle (Punuri et al. 2012), green coconut shell (Paul et al. 2014), etc., have been utilized for the synthesis of AuNPs. During our investigations on the utilization of triterpenoids (C30s) as renewable functional nano-entities (Bag et al. 2012; Bag and Dash 2011; Bag and Majumdar 2012; Bag and Paul 2012; Bag and Majumdar 2014), it occurred to us that the medicinally important bark extract

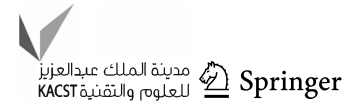


of Mimusops elengi, rich in polyphenolic compounds, can be utilized for the synthesis AuNPs from $\mathrm{HAuCl}_{4}$ (Baliga et al. 2011; Kadam et al. 2012).

Mimusops elengi (commonly known as Bakul) has been mentioned in all ancient scriptures of Ayurveda and has been used for medicinal purpose since centuries. The stem bark is dark gray in color, with striations and few cracks on the surface, internally reddish brown color having a bitter taste. The bark is used as a tonic, febrifuge, astringent, gargle for odontopathy, ulitis and ulemorrhagia (Baliga et al. 2011; Kadam et al. 2012). In the present study, the AuNPs have been synthesized using the bark extract of Mimusops elengi as a bio-reducing agent. The synthesized colloidal AuNPs were characterized by surface plasmon resonance spectroscopy, high-resolution transmission electron microscopy (HRTEM) and X-ray diffraction analysis.

Aminophenols are important precursors for the preparation of several analgesic and antipyretic drugs such as paracetamol, acetanilide and phenacetin. (Travis 2007). These compounds are also used as corrosion inhibitor in paints, anticorrosion lubricating agent in fuels, as photographic developer and in dye industries. Direct catalytic reduction of nitrophenols to aminophenols is of great significance to meet the current demand. In the present study, the catalytic activities of biosynthesized colloidal AuNPs have been investigated for the reduction of 3-nitrophenol (3-NP) and 4-nitrophenol (4-NP) in aqueous medium in the presence of sodium borohydride $\left(\mathrm{NaBH}_{4}\right)$ as a reducing agent. The kinetics of the reduction reactions investigated spectrophotometrically demonstrated that the colloidal AuNPs synthesized with lower concentration of plant extract were more efficient catalyst than that with higher concentration of plant extract.

\section{Materials and methods}

\section{Materials}

Au(III) solution

$\mathrm{HAuCl}_{4}$ was purchased from SRL (Sisco Research Laboratory) and used without further purification. $\mathrm{HAuCl}_{4}$ $(35.4 \mathrm{mg})$ was dissolved in distilled water $(10 \mathrm{~mL})$ to obtain a $10.42 \mathrm{mM} \mathrm{Au}(\mathrm{III})$ stock solution. 4-Nitrophenol and 3-nitrophenol were purchased from MERCK and LOBA Chemie, respectively.

\section{Preparation of the Mimusops elengi bark extract}

The bark of Mimusops elengi was collected from the Vidyasagar University campus. Dried and finely powdered
Mimusops elengi bark (50.0 g) was suspended in ethanol $(250 \mathrm{~mL})$ and stirred magnetically at room temperature for $1 \mathrm{~h}$ and then filtered. Volatiles of the brownish filtrate were removed under reduced pressure to afford a brown crystalline solid $(5.54 \mathrm{~g})$. The crude material $(0.660 \mathrm{~g})$ was purified by column chromatography (si-gel, 100-200 mesh) using $30 \%$ ethanol-ethyl acetate as the eluant affording a brownish shiny crystalline solid $(0.550 \mathrm{~g})$. The column purified bark extract $(0.0504 \mathrm{~g})$ was suspended in distilled water $(10 \mathrm{~mL})$ and sonicated in an ultra sonicator bath for $10 \mathrm{~min}$ to get a semi-transparent solution (5.04 $\left.\mathrm{g} \mathrm{L}^{-1}\right)$.

\section{Synthesis of nanoparticles}

Aliquots of $\mathrm{Au}$ (III) solution (0.16 mL, $10.42 \mathrm{mM}$ each) were added drop-wise to the bark extract solution to prepare a series of stabilized AuNPs where concentration of the bark extract varied from 50 to $600 \mathrm{mg} \mathrm{L}^{-1}$ and the concentration of $\mathrm{Au}(\mathrm{III})$ was fixed at $0.42 \mathrm{mM}$. UV-Vis spectroscopic measurements of the solutions were carried out after $3 \mathrm{~h}$ of $\mathrm{HAuCl}_{4}$ and Mimusops elengi bark extract was mixed.

\section{Characterization}

TEM images of AuNPs were recorded in JEOL JEM-2100 instrument. X-ray diffraction (XRD) analysis of the stabilized AuNPs was carried out in Rigaku Miniflex II diffractometer with $\mathrm{Cu}-\mathrm{K} \alpha$ radiation $(\lambda=1.54 \AA$ A). Mass spectra of the bark extract of Mimusops elengi were recorded in Shimadzu GCMS QP 2100 Plus. All the UVVis spectra were recorded in Shimadzu 1600 spectrophotometer.

\section{Results and discussion}

The presence of different types of plant secondary metabolites such as flavonoids, tannins, triterpenoids and saponins in the bark extract of Mimusops elengi has been reported (Baliga et al. 2011; Kadam et al. 2012; Rao et al. 2011). Indeed, mass spectral analysis of the bark extract of Mimusops elengi carried out in our laboratory indicated the presence of several polyphenolic compounds, steroids, etc. (supporting information Figure S1). Evidence for the presence of phenolic compounds in the bark extract of Mimusops elengi was also obtained from a positive ferric chloride test. The polyphenolic compounds present in the bark extract of Mimusops elengi can reduce $\mathrm{Au}(\mathrm{III})$ having a high reduction potential to $\mathrm{Au}(0)$ with concomitant oxidation of the polyphenolic compounds to the corresponding quinones. Collision of the $\mathrm{Au}(0)$ atoms with each 
other can yield gold nanoparticles (AuNPs) and the AuNPs can be stabilized by the resulting quinone derivatives and other coordinating ligands present in the bark extract. The stabilizing ligands present on the surface of the AuNPs will hinder further aggregation of the AuNPs. To test this, we treated increasing concentrations of the aqueous bark extract of Mimusops elengi contained in vials with $\mathrm{HAuCl}_{4}$ solution (Fig. 1). Visual observation of the appearance of pinkish red color almost instantly indicated the formation of AuNPs. On standing the vials at room temperature for several hours, intensification of the color was observed and then the intensities of the colloidal suspensions remained constant for several months indicating the stabilities of the synthesized AuNPs.

\section{UV-visible spectroscopy studies}

Due to charge transfer interactions between metal and the chloro ligands of $\mathrm{HAuCl}_{4}$, the UV-visible spectrum shows two peaks at 222 and $293 \mathrm{~nm}$ (Fig. 1). On reaction with increasing concentration of the Mimusops elengi bark extract, disappearance of these two peaks was observed with concomitant appearance of a surface plasmon resonance (SPR) band between 536 and $541 \mathrm{~nm}$ region. Variation of color of the AuNP colloids has been reported arising due to the change in size, shape, composition, crystallinity, etc. (Alkilany et al. 2013). In our present studies, the SPR band was observed from 536 to $541 \mathrm{~nm}$ region and the intensities increased with increasing concentration of the bark extract from $50 \mathrm{mg} \mathrm{L}^{-1}$ concentration to $600 \mathrm{mg} \mathrm{L}^{-1}$ concentration. The strong peaks around 274-279 nm

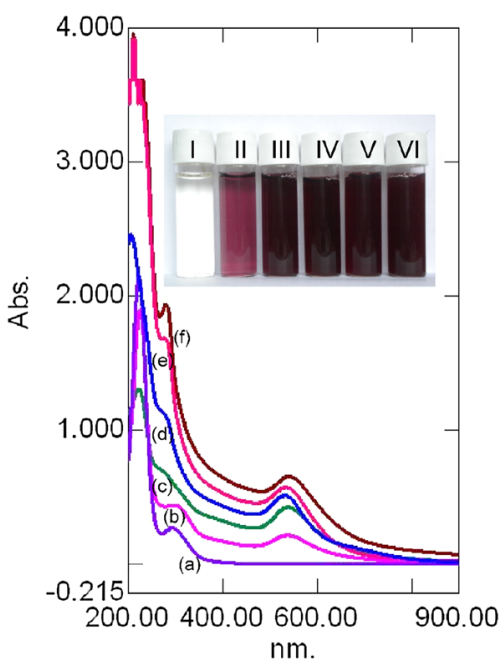

Fig. 1 UV-visible spectra of: $a \mathrm{HAuCl}_{4}(0.42 \mathrm{mM}), b-f$ AuNPs at $50,100,200,400,600 \mathrm{mg} \mathrm{L}^{-1}$ concentrations of Mimusops elengi bark extract, respectively. Inset photograph of the vials containing $I \mathrm{HAuCl}_{4}(0.42 \mathrm{mM}), I I-V I$ colloidal AuNPs stabilized by 50,100 , 200, 400, $600 \mathrm{mg} \mathrm{L}^{-1}$ concentrations of Mimusops elengi bark extract, respectively (after one and half hour of mixing) region at 400 and $600 \mathrm{mg} \mathrm{L}^{-1}$ concentration are due to the formation of quinone moieties formed due to the oxidation of the phenolic compounds by $\mathrm{Au}(\mathrm{III})$.

\section{HRTEM, EDX and XRD studies}

To study the size distribution, shape and morphology of the stabilized AuNPs synthesized from the bark extract of Mimusops elengi at varied concentration, high-resolution transmission electron microscopy (HRTEM) was performed (Fig. 2; see supporting information Figure S2). Mostly spherical-shaped AuNPs of 9-14 nm size embedded in organic matrix in esthetically pleasing patterns were observed (Fig. 2j, 1). The presence of the AuNPs held inside the organic matrix derived from the bark extract was clearly evident from Fig. 2d, e, g and h. Energy-dispersive $\mathrm{X}$-ray analysis (EDX) was performed to determine the elemental composition of the AuNPs synthesized and stabilized by the Mimusops elengi bark extract. Strong peaks of $\mathrm{Au}$ in the area-profile analysis of the synthesized nanoparticles (Fig. 2k) confirm the formation of AuNPs. The presence of organic matrix was also confirmed from the characteristic carbon peak. For, X-ray diffraction analysis, the colloidal AuNP samples were coated over a glass plate and the volatiles were removed under $150 \mathrm{~W}$ electric bulb. After the removal of the volatiles, the X-ray diffraction analysis was performed. The characteristic reflections of the planes (111), (200), (220) and (311) of the face-centered cubic AuNPs at $2 \theta=38.24^{\circ}, 44.45^{\circ}, 64.71^{\circ}$ and $77.78^{\circ}$, respectively (Figure S3) confirmed the formation of AuNPs. These values are in agreement with the reported standards JCPDS file no. 04-0784 for crystalline gold. The comparatively greater peak intensity of the (111) plane was observed which indicated the predominant orientation of the (111) plane.

\section{Mechanism of the formation of stabilized AuNPs}

The bark extract of Mimusops elengi is rich in different types of plant secondary metabolites such as flavonoids, tannins, triterpenoids and saponins. (Baliga et al. 2011; Kadam et al. 2012; Rao et al. 2011). Evidence for the presence of polyphenolic compounds was obtained from the ferric chloride test (supporting information). Mass spectral analysis of the bark extract carried out by us also supported the presence of the several polyphenolic compounds (Figure S1) including gallic acid $\left(\mathrm{M}^{+} 170\right)$, pinocembrin $\left(\mathrm{M}^{+} 256\right)$, quercetin $\left(\mathrm{M}^{+} 302\right)$, chlorogenic acid $\left(\mathrm{M}^{+} 354\right)$ or their analogs. A schematic representation of the possible mechanism for the formation of AuNPs and their stabilization by the polyphenolic compounds and other easily oxidizable compounds present in the bark

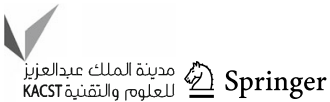



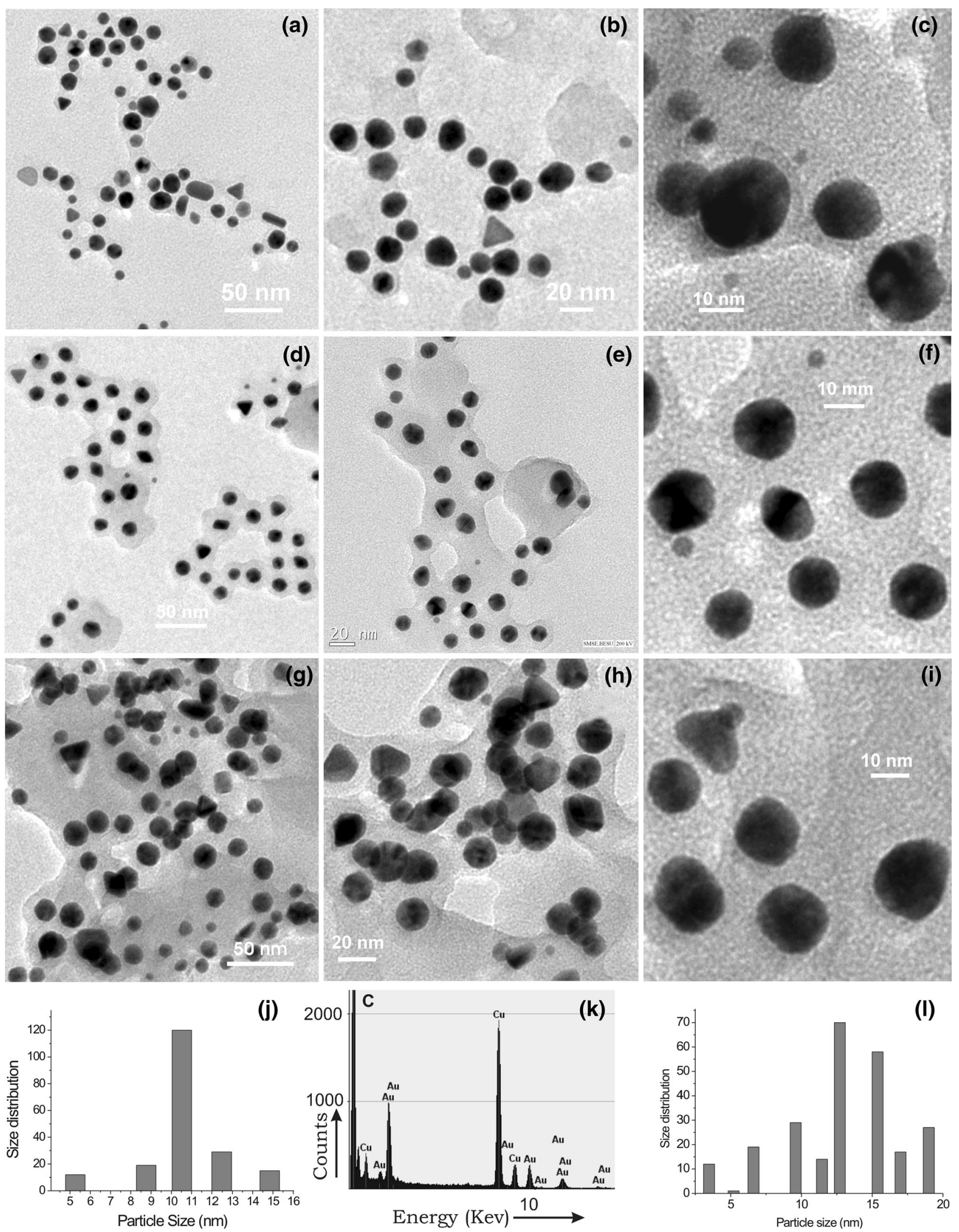

Fig. 2 a-c TEM images of AuNPs at $200 \mathrm{mg} \mathrm{L}^{-1}$, d-f TEM images of AuNPs at $400 \mathrm{mg} \mathrm{L}^{-1}$, $\mathbf{g}-\mathbf{i}$ TEM images of AuNPs at $600 \mathrm{mg} \mathrm{L}{ }^{-1}$, $\mathbf{j}$ histograms of AuNPs at $200 \mathrm{mg} \mathrm{L}^{-1}$, $\mathbf{k}$ EDX of AuNPs, $\mathbf{l}$ histogram of AuNPs at $400 \mathrm{mg} \mathrm{L}^{-1}$ 
extract is shown in Fig. 3. Initially, ortho-dihydroxy compounds can form a five-membered chelate complex with $\mathrm{Au}(\mathrm{III})$. Then, the chelated $\mathrm{Au}(\mathrm{III})$ can be reduced to $\mathrm{Au}(0)$ with concomitant oxidation of the ortho-dihydroxy compounds to quinones. Collision of the neighboring $\mathrm{Au}(0)$ atoms with each other leads to the formation of the AuNPs which can be stabilized by the polyphenolic compounds, quinones as well as the other coordinating phytochemicals present in the bark extract (Huang et al. 2010).

\section{Study of the catalytic activity of stabilized AuNPs}

To study the catalytic activity of the synthesized colloidal gold nanoparticles using the bark extract of Mimusops elengi, two model reactions were carried out: (a) the reduction of 3-nitrophenol $(0.05 \mathrm{mM})$ to 3-aminophenol and (b) 4-nitrophenol $(0.05 \mathrm{mM})$ to 4-aminophenol by sodium borohydride (13-14 $\mathrm{mM}$ ) at room temperature and the reactions were monitored by UV-visible spectroscopy (Fig. 4) (Wunder et al. 2011). The absorption band of 3 -nitrophenol at $331 \mathrm{~nm}$ shifted to $394 \mathrm{~nm}$ in the presence of sodium borohydride due to the formation of 3-nitrophenolate ion. No transformation was observed on standing the mixture at room temperature for several days. This is due to a very large kinetic barrier for the reduction reaction. Interestingly, on addition of bark extract stabilized AuNPs $\left(0.1 \mathrm{~mL}\right.$, synthesized with $100 \mathrm{mg} \mathrm{L}^{-1}$ bark extract of Mimusops elengi) to the mixture, lowering of the intensity of the absorption peak at $394 \mathrm{~nm}$ was observed indicating the formation of 3-aminophenol. Complete disappearance of the $394 \mathrm{~nm}$ peak was observed within $300 \mathrm{~s}$ indicating completion of the reduction demonstrating the excellent catalytic activity of the synthesized colloidal AuNPs from the Mimusops elengi bark extract. The change in intensity of the absorption maxima of 3-nitrophenolate in alkaline medium (at $394 \mathrm{~nm}$ ) with time provides a reasonable tool for the study of its reduction kinetics. As the concentration of $\mathrm{BH}_{4}^{-}$was much higher (approximately 300 fold) than that of 3-nitrophenol, its concentration remained practically constant during the reduction reaction and the reaction can be assumed as a pseudo-first-order reaction. A good linear correlation between $\ln \left(A / A_{0}\right)$ vs time was obtained (supporting information Figure S4a) and the value of the catalytic rate constant $(k)$ was calculated to be $7.33 \times 10^{-3} \mathrm{~s}^{-1}$. Interestingly, on addition of double amount of stabilized AuNPs ( $0.2 \mathrm{~mL}$, synthesized with $100 \mathrm{mg} \mathrm{L}^{-1}$ bark extract of Mimusops elengi) to the mixture, the rate of the reduction reaction became much faster and the $394 \mathrm{~nm}$ peak completely disappeared within $30 \mathrm{~s}$ with concomitant formation of 3-aminophenol. Due to the very fast reaction, the catalytic rate constant could not be calculated for this reduction reaction. Increase in rates of the reduction reactions with increasing volume of the catalyst is due to the presence of larger number of reaction sites. Moreover, the rate of reduction reactions also became much faster when we used the AuNPs $(0.2 / 0.1 \mathrm{~mL})$ stabilized with $50 \mathrm{mg} \mathrm{L}^{-1}$ bark extract of Mimusops elengi (Table 1) and the reduction was complete within $30 \mathrm{~s}$.

Similarly, the absorption band of 4-nitrophenol at $319 \mathrm{~nm}$ was shifted to $401 \mathrm{~nm}$ after the addition of sodium borohydride due to the formation of 4-nitrophenolate ion. Here, also no further transformation was occurred on keeping the reaction mixture at room temperature due to the very large kinetic barrier of the reduction. Interestingly, on addition of bark extract stabilized AuNPs $\left(0.2 \mathrm{~mL}\right.$, synthesized with $50 \mathrm{mg} \mathrm{L}^{-1}$ bark extract of Mimusops elengi) to the mixture, lowering of the intensity of the absorption peak at $401 \mathrm{~nm}$ was observed indicating the formation of 4-aminophenol. Complete disappearance of the $401 \mathrm{~nm}$ peak was observed within $480 \mathrm{~s}$ indicating completion of the reduction demonstrating the excellent catalytic activity of the

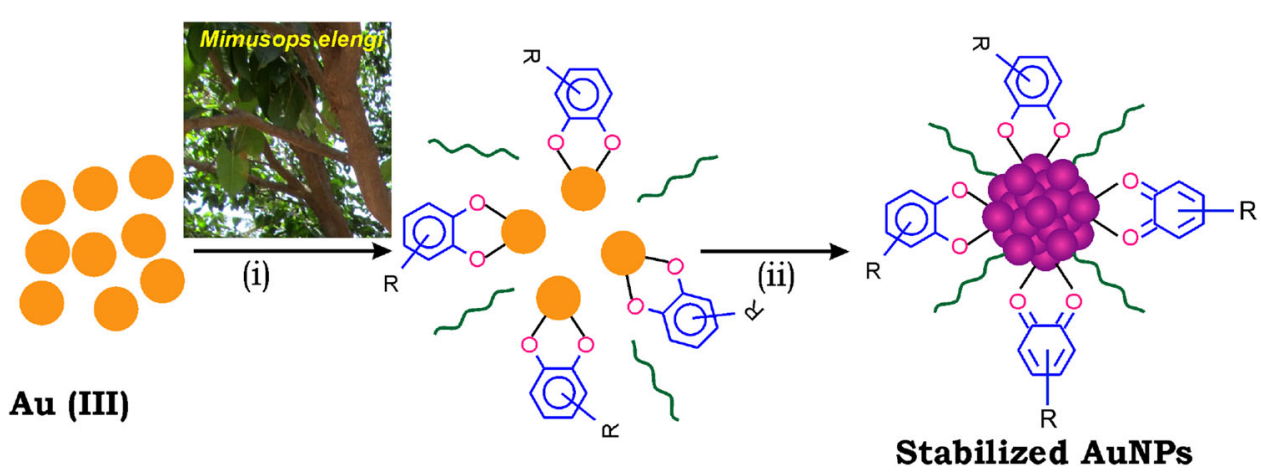

Fig. 3 Mechanism of formation and stabilization of gold nanoparticles by polyphenolic compounds and other plant metabolites (shown by green curved line) present in the bark extract of Mimusops elengi: $i$ five-membered chelate ring formation by the polyphenolic

compounds of bark extract with $\mathrm{Au}(\mathrm{III}), i i$ auto-reduction and stabilization by the polyphenolic compounds, quinones and other coordinating plant metabolites 


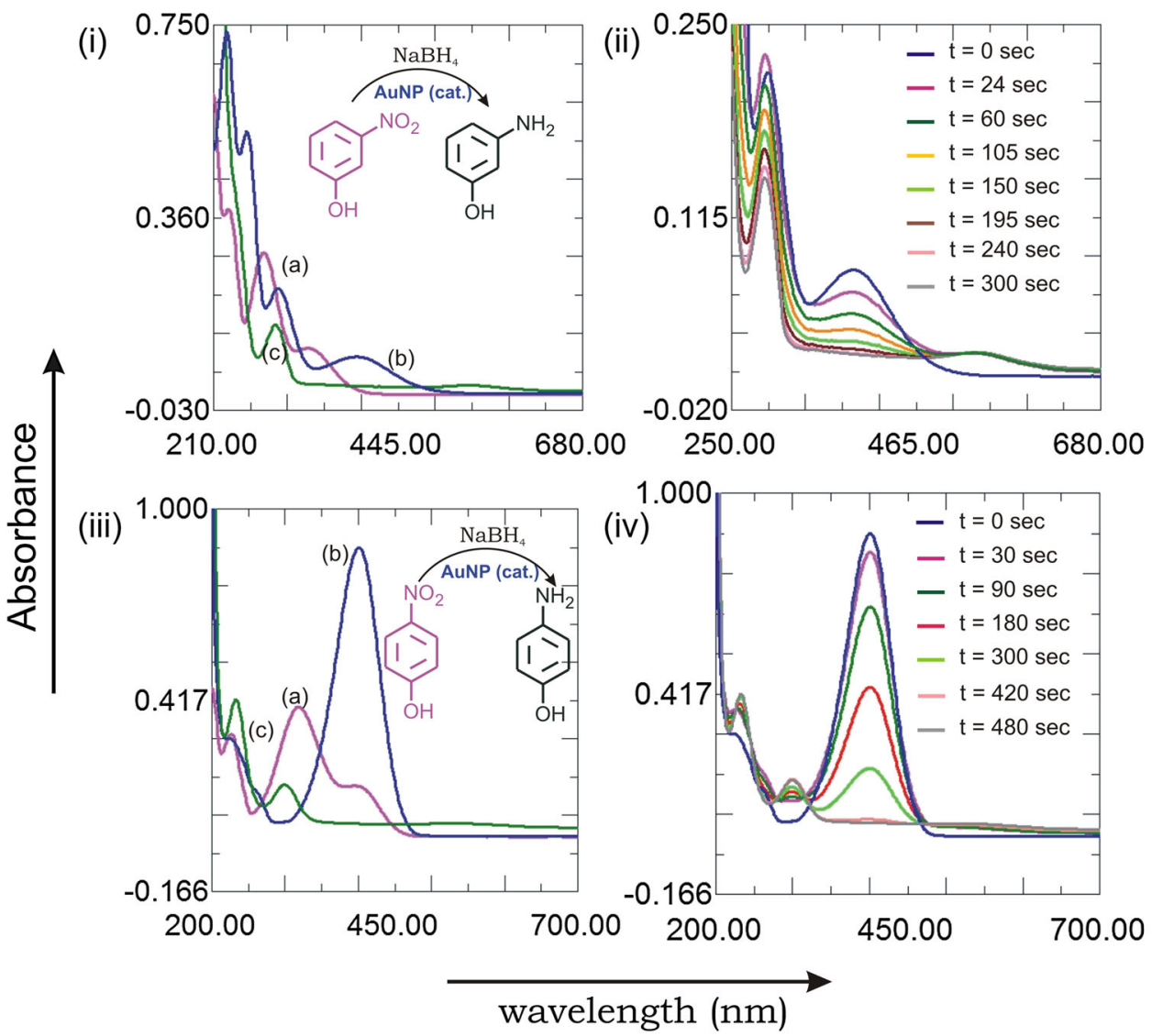

Fig. 4 i, ii UV-visible spectra during the catalytic reduction of 3-nitrophenol to 3-aminophenol using stabilized AuNPs (synthesized with $100 \mathrm{mg} \mathrm{L}^{-1}$ bark extract): i UV-visible spectra of $a$ aqueous 3-nitrophenol solution $(0.05 \mathrm{mM}), b$ aqueous 3-nitrophenol solution $(0.05 \mathrm{mM})$ in the presence of added sodium borohydride $(15 \mathrm{mM})$, $c$ after complete reduction of 3-nitrophenol using $0.1 \mathrm{ml}$ of colloidal AuNPs as catalyst. ii Catalytic reduction of 3-nitrophenolate to 3-aminophenol at different time intervals using $0.1 \mathrm{ml}$ stabilized AuNPs; iii, iv UV-visible spectra during the catalytic reduction of 4-nitrophenol to 4-aminophenol using stabilized AuNPs (synthesized with $50 \mathrm{mg} \mathrm{L}^{-1}$ bark extract): iii UV-visible spectra of $a$ aqueous 4-nitrophenol solution ( $0.05 \mathrm{mM}), b$ aqueous 4-nitrophenol solution $(0.05 \mathrm{mM})$ in the presence of added sodium borohydride $(15 \mathrm{mM})$, $c$ after complete reduction of 4-nitrophenol using $0.2 \mathrm{ml}$ of colloidal AuNPs as catalyst. iv Catalytic reduction of 4-nitrophenolate to 4-aminophenol at different time intervals using $0.2 \mathrm{ml}$ stabilized AuNPs

Table 1 Catalytic activity of the Mimusops elengi bark extract stabilized AuNPs in the reduction of 3- and 4-nitrophenol by sodium borohydride

\begin{tabular}{|c|c|c|c|c|c|c|}
\hline \multirow[t]{2}{*}{ Entry } & \multirow{2}{*}{$\begin{array}{l}\text { Substrate } \\
(\mathrm{mM})\end{array}$} & \multirow{2}{*}{$\begin{array}{l}\mathrm{NaBH}_{4} \\
(\mathrm{mM})\end{array}$} & \multicolumn{2}{|c|}{ Colloidal AuNPs used } & \multirow{2}{*}{$\begin{array}{l}\text { Reaction } \\
\text { completion } \\
\text { time (s) }\end{array}$} & \multirow{2}{*}{$\begin{array}{l}\text { Catalytic rate constant } \\
(k)\left(\mathrm{s}^{-1}\right)\end{array}$} \\
\hline & & & $\begin{array}{l}\text { Volume } \\
(\mathrm{mL})\end{array}$ & $\begin{array}{l}\text { Conc. of bark extract } \\
\text { used }\left(\mathrm{mg} \mathrm{L}^{-1}\right)\end{array}$ & & \\
\hline 1 & $3-\mathrm{NP} / 0.05$ & 13.5 & 0.2 & 50 & $<30$ & Can't be calculated \\
\hline 2 & $3-\mathrm{NP} / 0.05$ & 13.9 & 0.1 & 50 & $<30$ & Can't be calculated \\
\hline 3 & $3-\mathrm{NP} / 0.05$ & 13.5 & 0.2 & 100 & $<30$ & Can't be calculated \\
\hline 4 & $3-\mathrm{NP} / 0.05$ & 13.9 & 0.1 & 100 & 300 & $7.33 \times 10^{-3}$ \\
\hline 5 & $4-\mathrm{NP} / 0.05$ & 13.5 & 0.2 & 50 & 480 & $6.53 \times 10^{-3}$ \\
\hline 6 & $4-\mathrm{NP} / 0.05$ & 13.5 & 0.2 & 100 & No reduction until $600 \mathrm{~s}$ & \\
\hline
\end{tabular}

synthesized colloidal AuNPs from the Mimusops elengi bark extract. In this case also, a good linear correlation between $\ln \left(A_{\mathrm{t}} / A_{0}\right)$ vs time was obtained (supporting information Figure $S 4 b$ ) and the value of the catalytic rate constant $(k)$ was calculated to be $6.53 \times 10^{-3} \mathrm{~s}^{-1}$. The observed rate constants were similar to that observed by us previously and others on similar systems (Majumdar and Bag 2013; Gangula et al. 2011). 
During the course of the reactions, it was observed that the colloidal AuNPs synthesized and stabilized with 50 $\mathrm{mg} \mathrm{L}^{-1}$ bark extract of Mimusops elengi were more efficient catalyst than the colloidal AuNPs synthesized and stabilized with $100 \mathrm{mg} \mathrm{L}^{-1}$ bark extract of Mimusops elengi. In the case of 3-nitrophenol reduction, with $50 \mathrm{mg} \mathrm{L}^{-1}$ bark extract stabilized AuNPs $(0.1 \mathrm{~mL})$, the complete reduction occurred within $30 \mathrm{~s}$ whereas with $100 \mathrm{mg} \mathrm{L}^{-1}$ bark extract stabilized AuNPs $(0.1 \mathrm{~mL})$, the complete reduction occurred within $300 \mathrm{~s}$. Similarly, for 4-nitrophenol reduction, with the $50 \mathrm{mg} \mathrm{L}^{-1}$ bark extract stabilized AuNPs $(0.2 \mathrm{~mL})$, the complete reduction occurred within $480 \mathrm{~s}$ but with the $100 \mathrm{mg} \mathrm{L}^{-1}$ bark extract stabilized AuNPs $(0.2 \mathrm{~mL})$, no reduction was observed even after $10 \mathrm{~min}$.

It is believed that, both 3-nitrophenol/4-nitrophenol and the reducing agent $\mathrm{BH}_{4}{ }^{-}$are adsorbed on the surface of the AuNPs and the surface hydride ions are then transferred to 3-nitrophenol/4-nitrophenol thereby facilitating the reduction reaction (Wunder et al. 2011). In both reductions of 3-nitrophenol and 4-nitrophenol, the rates were slower with AuNPs synthesized with $100 \mathrm{mg} \mathrm{L}^{-1}$ concentrations of plant extract than that with $50 \mathrm{mg} \mathrm{L}^{-1}$ concentration. This may be due to the fact that large excess of the plant extracts surrounding the AuNPs may sterically hinder the AuNPs for catalytic activity.

\section{Conclusions}

A very mild and efficient method for the green synthesis of colloidal gold nanoparticles has been demonstrated using the bark extract of medicinally important Mimusops elengi. According to our knowledge, this is the first report of the synthesis of AuNPs using Mimusops elengi bark extract. The polyphenolic compounds present in the bark extract acts as an effective reducing agent as well as stabilizing agent and AuNPs of 9-14 nm size were formed. The application of the synthesized colloidal AuNPs has been demonstrated using it as efficient catalyst for the reduction of 3-nitrophenol to 3-aminophenol as well as 4-nitrophenol to 4-aminophenol at room temperature in aqueous medium. As the aminophenols are important precursors for the preparation of many industrially useful compounds, direct catalytic reduction of nitrophenols to aminophenols described here has tremendous significance for its industrial applications.

Acknowledgments We thank CSIR [02(0068)/12/EMR-II], UGCSAP and DST-FIST New Delhi, for financial support and infrastructural facilities. RM thanks UGC, New Delhi, for a research fellowship.

Open Access This article is distributed under the terms of the Creative Commons Attribution 4.0 International License (http:// creativecommons.org/licenses/by/4.0/), which permits unrestricted use, distribution, and reproduction in any medium, provided you give appropriate credit to the original author(s) and the source, provide a link to the Creative Commons license, and indicate if changes were made.

\section{References}

Alkilany AM, Lohse SE, Murphy CJ (2013) The gold standard: gold nanoparticle libraries to understand hydrogenation catalyst. Acc Chem Res 46:650-661

Anastas PT, Kirchhoff MM (2002) Origins, current status, and future challenges of green chemistry. Acc Chem Res 35:686-694

Aslan K, Perez-Luna VH (2002) Surface modification of colloidal gold by chemisorption of alkanethiols in the presence of a nonionic surfactant. Langmuir 18:6059-6065

Bag BG, Dash SS (2011) First self-assembly study of betulinic acid, a renewable nano-sized, 6-6-6-6-5 pentacyclic monohydroxy triterpenic acid. Nanoscale 3:4564-4566

Bag BG, Majumdar R (2012) Self-assembly of a renewable nanosized triterpenoid 18ß-glycyrrhetinic acid. RSC Adv 2:8623-8626

Bag BG, Majumdar R (2014) Vesicular self-assembly of a natural triterpenoid arjunolic acid in aqueous medium: study of entrapment properties and in situ generation of gel-gold nanoparticle hybrid material. RSC Adv 4:53327-53334

Bag BG, Paul K (2012) Vesicular and fibrillar gels by self-assembly of nanosized oleanolic acid. Asian J Org Chem 1:150-154

Bag BG, Garai C, Majumdar R, Laguerre M (2012) Natural triterpenoids as renewable nanos. Struct Chem 23:393-398

Baliga MS, Pai RJ, Bhat HP, Palatty PL, Boloor R (2011) Chemistry and medicinal properties of the Bakul (Mimusops elengi Linn): a review. Food Res Int 44:1823-1829

Das S, Bag BG, Basu R (2014) Abroma augusta Linn bark extract mediated green synthesis of gold nanoparticles and its application in catalytic reduction. Appl Nanosci. doi:10.1007/s13204014-0384-4

Dash SS, Bag BG, Hota P (2014a) Lantana camara Linn leaf extract mediated green synthesis of gold nanoparticles and study of its catalytic activity. Appl Nanosci. doi:10.1007/s13204-014-0 323-4

Dash SS, Majumdar R, Sikder AK, Bag BG, Patra BK (2014b) Saraca indica bark extract mediated green synthesis of polyshaped gold nanoparticles and its application in catalytic reduction. Appl Nanosci 4:485-490

Fujiwara H, Yanagida S, Kamat PV (1999) Visible laser induced fusion and fragmentation of thionicotinamide-capped gold nanoparticles. J Phys Chem B 103:2589-2591

Gangula A, Podila R, Ramakrishna M, Karanam L, Janardhana C, Rao AM (2011) Catalytic reduction of 4-nitrophenol using biogenic gold and silver nanoparticles derived from Breynia rhamnoides. Langmuir 27:15268-15274

Huang X, Wu H, Liao X, Shia B (2010) One-step, size-controlled synthesis of gold nanoparticles at room temperature using plant tannin. Green Chem 12:395-399

Iravani S (2011) Green synthesis of metal nanoparticles using plants. Green Chem 13:2638-2650

Kadam PV, Yadav KN, Deoda RS, Shivatare RS, Patil MJ (2012) Mimusops elengi: a review on ethnobotany, phytochemical and pharmacological profile. J Pharm Phytochem 1:64-74

Majumdar R, Bag BG (2013) Acacia nilotica (Babool) leaf extract mediated size controlled rapid synthesis of gold nanoparticles and study of its catalytic activity. Int Nano Lett 3:53 
Mie G (1908) Contributions to the optics of cloudy media, particularly of colloidal metal solutions. Ann Phys 25:377-445

Murphy CJ, Gole AM, Stone JW, Sisco PN, Alkilany AM, Goldsmith EC, Baxter SC (2008) Gold nanoparticles in biology: beyond toxicity to cellular imaging. Acc Chem Res 41:1721-1730

Paul K, Bag BG, Samanta K (2014) Green coconut (Cocos nucifera Linn) shell extract mediated size controlled green synthesis of polyshaped gold nanoparticles and its application in catalysis. Appl Nanosci 4:769-775

Prati L, Villa A (2014) Gold colloids: from quasi-homogeneous to heterogeneous catalytic systems. Acc Chem Res 47:855-863

Punuri JB, Sharma P, Sibyala S, Tamuli R, Bora U (2012) Piper betlemediated green synthesis of biocompatible gold nanoparticles. Int Nano Lett 2:18

Rao KS, Munjuluri PR, Keshar NK (2011) Invitro antioxidant activity and total phenolic content of Mimusops elengi bark. Ind J Pharm Edu Res 45:317-323
Thomas KG, Kamat PV (2003) Chromophore-functionalized gold nanoparticles. Acc Chem Res 36:888-898

Travis AS (2007) Manufacture and uses of the anilines: a vast array of processes and products. In: Rappoport Z (ed) The chemistry of anilines, part 1. Wiley, New York, pp 715-782

Weisbecker CS, Merritt MV, Whitesides GM (1996) Molecular selfassembly of aliphatic thiols on gold colloids. Langmuir 12:3763-3772

Wunder S, Lu Y, Albrecht M, Ballauff M (2011) Catalytic activity of faceted gold nanoparticles studied by a model reaction: evidence for substrate-induced surface restructuring. ACS Catal 1:908-916

Zhang Y, Cui X, Shi F, Deng Y (2012) Nano-gold catalysis in fine chemical synthesis. Chem Rev 112:2467-2505 